\title{
Race is not prognostic in unfavorable prostate cancer: an NCDB analysis
}

Joyson Kodiyan, MD*, Mark Ashamalla, MD, Adel Guirguis, MBBCh, MS, Hani Ashamalla, MD, FCCP

\begin{abstract}
Introduction: It is commonly held that race serves as a significant prognosticator in prostate cancer. We sought to analyze whether race impacted overall survival $(\mathrm{OS})$ of men receiving standard of care treatment.

Materials and Methods: All data was obtained from the NCDB (National Cancer Database) and initially contained 1,294,126 cases of prostate cancer diagnosed between 2004 and 2013. Patients were excluded if they had metastatic or nodal disease, received chemotherapy, or had noninvasive disease. Patients were grouped into 2 cohorts as per NCCN (National Comprehensive Cancer Network) criteria: favorable risk and unfavorable risk. All patients received risk-appropriate radiation therapy or surgery, and were subsequently analyzed. Black and white men were also matched 1:1 within each risk cohort using propensity scores, and multivariate analysis was conducted on these matched cohorts.

Results: The final cohort 77,448 patients. Median follow-up 58.7 months (range, 48-143.5). In the favorable risk cohort, white men had superior OS compared with black men, $77.63 \%$ versus $80.57 \%$ at 10 years (hazard ratio, 0.644; 95\% confidence interval, 0.536-0.773; $P<0.001)$. In the unfavorable cohort, race was not a significant prognostic factor for OS on unmatched analysis; 10 -year survival $73.6 \%$ and $71.3 \%$ (hazard ratio, $0.941 ; 95 \%$ confidence interval, $0.848-1.044 ; P=0.249$ ), and significant interaction existed with age. Results were unchanged in propensity score matched cohorts.

Conclusion: The prognostic value of race-related biological differences of prostate cancer may hold less value in higher risk disease than is commonly believed.
\end{abstract}

Keywords: Prostate, Cancer, Race

It is commonly held that race serves as a significant prognosticator in prostate cancer. Several retrospective series have demonstrated that black men with low risk (LR) prostate adenocarcinoma experience more rapid disease progression when placed on active surveillance (AS) and require subsequent definitive therapy in comparison to white men ${ }^{[1-3]}$. These differences have previously been theorized to be due to differences in tumor biology, such as tumor volume/prostate-specific antigen (PSA)

Department of Radiation Oncology, NewYork-Presbyterian Brooklyn Methodist Hospital Radiation Oncology, Brooklyn, NY

This manuscript has been peer reviewed.

Sponsorships or competing interests that may be relevant to content are disclosed at the end of this article.

${ }^{*}$ Corresponding author. Address: Department of Radiation Oncology, NewYorkPresbyterian Brooklyn Methodist Hospital Radiation Oncology, 506 6th Street, Brooklyn, NY 11215. Tel.: (+ 718) 780-3677; fax: (+ 718) 780-3677. E-mail address: jok9122@nyp.org (J. Kodiyan).

Copyright @ 2020 The Authors. Published by Wolters Kluwer Health, Inc. on behalf of IJS Publishing Group Ltd. This is an open-access article distributed under the terms of the Creative Commons Attribution-Non Commercial-No Derivatives License 4.0 (CCBY-NC-ND), where it is permissible to download and share the work provided it is properly cited. The work cannot be changed in any way or used commercially without permission from the journal.

International Journal of Surgery Oncology (2020) 5:e86

Received 3 January 2020; Accepted 3 February 2020

Published online 3 April 2020

Supplemental Digital Content is available for this article. Direct URL citations appear in the printed text and are provided in the HTML and PDF versions of this article on the journal's website, www.IJSOncology.com.

http://dx.doi.org/10.1097//J9.0000000000000086 ratio, Gleason score (GS) $\leq 6$ and expression of several biomarkers ${ }^{[2,3]}$. Several studies have demonstrated that black men have more aggressive prostate cancer that transforms earlier and presents at a younger age compared with white men ${ }^{[4,5]}$, and are more often to have adverse features seen on pathology after radical prostatectomy $(\mathrm{RP})^{[6]}$. Black men are also more likely to present with higher PSA and greater tumor burden than white men, which has been observed in a population of patients with equal access to care and screening ${ }^{[7]}$. In a matched analysis of a small cohort of T1c patients, black patients demonstrated significantly greater pathologic GS upstaging and tumor volume/ PSA ratio than their white counterparts ${ }^{[8]}$.

Socioeconomic factors and lack of access may be underlying these perceived differences between races $^{[7,9]}$. An NCDB (National Cancer Database) analysis demonstrated that white men were more likely to receive definitive therapy for their prostate cancer compared with black men, which remained significant in subgroup analysis based on geographical region and facility type ${ }^{[9]}$.

Given the increasing evidence of significant differences in the biology and genetics of prostate cancer between black and white men, we sought to analyze whether race impacted survival of patients receiving appropriate standard of care treatment regimens using a large national database.

\section{Materials and methods}

All data was obtained from the NCDB, a cancer database derived from hospital registry data and sponsored by the American Cancer Society and the American College of Surgeons. The 
database contains $>70 \%$ of new cancer diagnoses made in the United States.

The database initially contains 1,294,126 cases of prostate cancer cases diagnosed between 2004 and 2013. Only black and white men were analyzed in this study. All analyzed patients had a minimum follow-up of 48 months with documented follow up time. Patients were eliminated from the study if they had metastatic or nodal disease, received chemotherapy, or had noninvasive disease (Fig. 1). Patients receiving external beam radiotherapy (EBRT) must have received at least $7560 \mathrm{cGy}$. Patients were included only if they received risk-appropriate definitive therapy as described below. Those receiving surgery were allowed to receive adjuvant androgen deprivation therapy (ADT) or adjuvant EBRT.

Patients were first risk-stratified according to NCCN (National Comprehensive Cancer Network) criteria using clinical GS, PSA, and clinical T-stage. Patients were subsequently grouped into 2 broad cohorts: favorable and unfavorable risk. The favorable risk cohort included LR and favorable intermediate risk. Treatment modalities included RP with or without pelvic lymph node dissection (PLND), EBRT, or brachytherapy alone (BT).

For the unfavorable risk cohort, which consisted of unfavorable intermediate risk, high risk, and very high risk, treatment modalities included RP with PLND, EBRT + ADT, or $\mathrm{EBRT}+\mathrm{BT}+\mathrm{ADT}$. Patients receiving surgery (RP alone or RP with PLND) were further classified into those receiving RT, ADT, or both RT and ADT as well.

The endpoint of interest was overall survival (OS) which was measured from the date of diagnosis to date of death; observations that were alive at time of last follow-up were censored. Black and white men were matched 1:1 within each risk category using calculated propensity scores.

Propensity score calculations utilized the following covariates: treatment received, facility type, facility location, time from diagnosis to initiation of treatment, insurance status, income level, comorbidity status based on the Charleson-Deyo score,

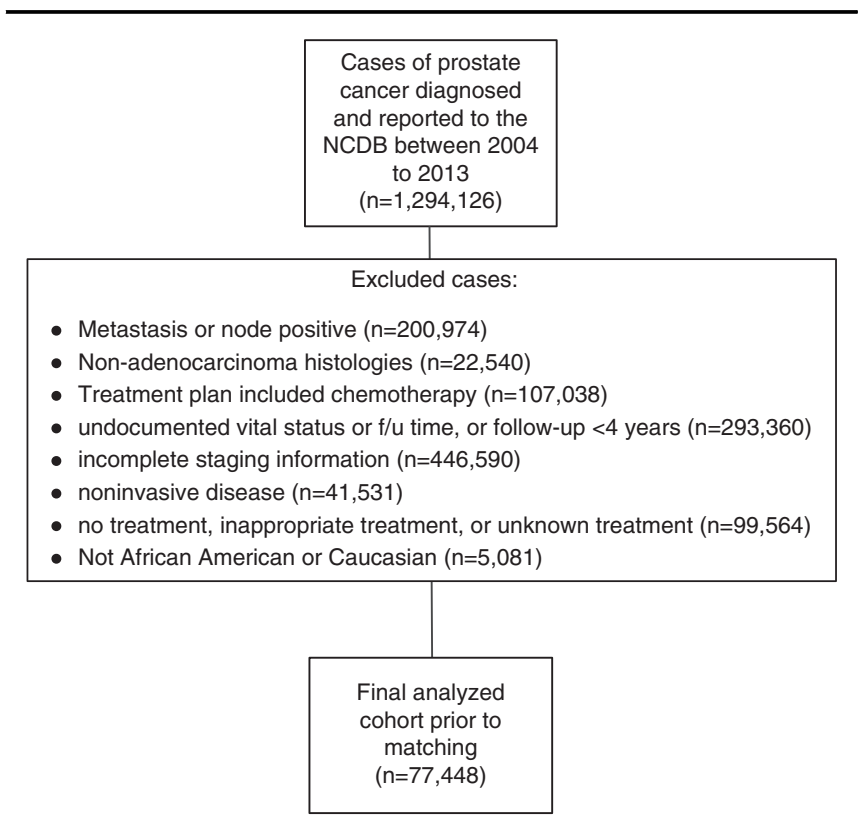

Figure 1. Consort diagram.
NCCN risk group, GS, PSA, clinical T-stage, and age. Descriptive statistics were calculated using $\chi^{2}$ analysis for each race in each risk cohort. Balance of cohorts was assessed using standardized mean differences of each covariate, with values $<10 \%$ indicating a sufficiently matched cohort ${ }^{[10]}$. Kaplan-Meier survival curves were constructed and analyzed using the log-rank test on matched cohorts. All Cox proportional hazards models were constructed using variables selected a priori on unmatched cohorts and propensity score-matched cohorts (PS-MVA). Interaction testing was done between covariates and race, and those covariates with significant interactions were subsequently analyzed in stratified multivariate analysis.

A sensitivity analysis was done for both favorable and unfavorable cohorts. The first was done by stratifying those receiving RP by whether the technique was robotic or not, controlling for surgical technique which may have significantly influenced outcomes ${ }^{[11]}$. A second sensitivity analysis was done in the unfavorable risk cohort stratifying patients that received RP by weather adjuvant ADT and/or RT was used. Treatment groups were as follows: EBRT + ADT, EBRT + BT + ADT, RP with PLND, RP with PLND + ADT, RP with PLND + RT, and RP with $\mathrm{PLND}+\mathrm{ADT}+\mathrm{RT}$. All $P$-values were 2 -sided using a significance level of 0.05 . Statistical analysis was done using $\mathrm{R}$ version 3.5.1.

\section{Results}

\section{Patients characteristics among risk groups}

The final analyzed cohort included 77,448 patients (Fig. 1) with a median follow-up time of 58.7 months (range, 48-143.5 mo). All covariates in these unmatched cohorts demonstrated significant imbalances between black and white men (Tables A1, Supplemental Digital Content 1, http://links.lww.com/IJSO/A5, A5, Supplemental Digital Content 5, http://links.lww.com/IJSO/ A9). All covariates in propensity score-matched cohorts had standardized mean differences $<10 \%$, denoting a well-balanced match (Tables A2, Supplemental Digital Content 2, http://links. lww.com/IJSO/A6, A6, Supplemental Digital Content 6, http:// links.lww.com/IJSO/A10).

In the favorable risk cohort, 3724 white men were matched to 3724 black men (Table A2, Supplemental Digital Content 2, http://links.lww.com/IJSO/A6). In the unfavorable risk cohort, 2543 white men were matched to 2543 black men (Table A6, Supplemental Digital Content 6, http://links.lww.com/IJSO/ A10).

\section{OS and effect of race}

In the favorable cohort, white men had superior OS compared with black men, $77.63 \%(74.31-81.10)$ versus $80.57 \%$ [95\% confidence interval (CI), 74.21-87.47] at 10 years [hazard ratio (HR), 0.644; 95\% CI, 0.536-0.773; $P<0.001$, Table 1], which was also observed on PS-MVA (HR, 0.665; 95\% CI, 0.498-0.887; $P<0.006$, Table 1). The other significant predictors for improved OS in the favorable cohort was being treated at an academic/research facility, Charleson-Deyo comorbidity score score of 0 compared with 1 or $2+$, or younger age (Table A3, Supplemental Digital Content 3, http://links.lww.com/IJSO/ A7). White men continued to have a survival advantage in the sensitivity analysis accounting for adjuvant therapies (ADT or EBRT) in those having had an RP (HR, 0.647; 95\% CI, 
Table 1

HRs and Cls for whites (compared with blacks) in unmatched and PSM cohorts.

\begin{tabular}{lcrrrr}
\hline & \multicolumn{2}{c}{ Unmatched } & & \multicolumn{2}{c}{ PSM } \\
\cline { 2 - 3 } \cline { 6 - 6 } Risk & HR (95\% Cl) & \multicolumn{1}{c}{$\boldsymbol{P}$} & & HR (95\% Cl) & $\boldsymbol{P}$ \\
\hline Favorable & $0.644(0.536-0.773)$ & $<0.001$ & & $0.665(0.498-0.887)$ & 0.006 \\
Unfavorable & $0.941(0.848-1.044)$ & 0.249 & & $0.967(0.815-1.148)$ & 0.703 \\
\hline
\end{tabular}

$\mathrm{Cl}$ indicates confidence interval; $\mathrm{HR}$, hazard ratio; PSM, propensity score matched.

$0.538-0.777 ; P<0.001$, Table 2 ). Sensitivity analysis controlling for surgical approach (open, laparoscopic or robotic) also demonstrated that white race was a significant predictor for improved survival (HR, 0.644; $95 \%$ CI, $0.536-0.774 ; P<0.001$, Table 3). Income bracket was the only variable found to significantly interact with race and survival, and subgroup analysis demonstrated that in the lowest and highest bracket subgroups, white race was a significant predictor for improved survival (Table A4, Supplemental Digital Content 4, http://links.lww. com/IJSO/A8).

In the unfavorable cohort, race was not found to be a significant prognostic factor for OS on unmatched analysis; 10 -year survival was $73.6 \%(95 \% \mathrm{CI}, 72.4 \%-74.7 \%)$ and $71.3 \%(95 \%$ CI, 68.6-74.2) in white and black men, respectively (HR, 0.941; $95 \%$ CI, 0.848-1.044; $P=0.249$, Table 1). Race was also not a significant predictor for OS on PS-MVA (HR, 0.967; 95\% CI, 0.815-1.148; $P=0.703$, Table 1). Both EBRT + BT + ADT (HR, $0.628 ; 95 \% \mathrm{CI}, 0.461-0.856 ; P=0.003$ ) and RP + PLND (HR, $0.622 ; 95 \%$ CI, $0.493-0.784 ; P<0,001)$ were superior to EBRT + ADT (Table A7, Supplemental Digital Content 7, http:// links.lww.com/IJSO/A11). Charleson-Deyo score of 0 predicted for improved OS compared with 1 or $2+$ (Table A7, Supplemental Digital Content 7, http://links.lww.com/IJSO/ A11). Sensitivity analysis controlling for receipt of adjuvant ADT or EBRT continued to demonstrate no significant difference in survival between white and black men (HR, 0.949; 95\% CI, $0.856-1.053 ; P=0.324$, Table 4). Similarly, when controlling for surgical approach, no significant difference was seen between black and white men (HR, 0.942; 95\% CI, 0.849-1.040; $P=0.256$, Table 5). Significant interaction was seen between age

\section{Table 2}

Sensitivity analysis, favorable risk cohort, stratifying by adjuvant treatment after surgery.

\begin{tabular}{lcr}
\hline Variables & HR (95\% Cl) & $\boldsymbol{P}$ \\
\hline Race & Reference & \\
$\quad$ Black & $0.647(0.538-0.777)$ & $<0.001$ \\
$\quad$ White & Reference & \\
Treatment & $1.110(0.906-1.350)$ & 0.322 \\
BT & $0.880(0.599-1.291)$ & 0.512 \\
EBRT & $0.640(0.530-0.773)$ & $<0.001$ \\
RP + ADT & $0.751(0.278-2.031)$ & 0.573 \\
RP, no ADT & $1.010(0.602-1.690)$ & 0.975 \\
RP + RT + ADT & & \\
RP + RT, no ADT &
\end{tabular}

ADT indicates androgen deprivation therapy; $\mathrm{BT}$, brachytherapy; $\mathrm{Cl}$, confidence controlled for program, facility, geography, income, age comorbidity score, clinical T stage, clinical Gleason score, PSA and treatment delay interval; EBRT, external beam radiotherapy; HR, hazard ration; PLND, pelvic lymph node dissection; RP, radical prostatectomy; RT, radiotherapy.
Table 3

Sensitivity analysis stratifying favorable risk cohort by surgical approach.

\begin{tabular}{lcc}
\hline Variables & HR $\mathbf{( 9 5 \%}$ Cl) & $\boldsymbol{P}$ \\
\hline Race & Reference & \\
$\quad$ Black & $0.644(0.536-0.774)$ & $<0.001$ \\
$\quad$ White & Reference & \\
Treatment & $1.099(0.901-1.341)$ & \\
BT & $0.862(0.546-1.360)$ & 0.352 \\
EBRT & $1.245(0.308-5.031)$ & 0.522 \\
RP-laparascopic & $0.642(0.487-0.845)$ & 0.758 \\
RP-open & $0.560(0.229-1.369)$ & 0.002 \\
RP-robotic & $0.633(0.475-0.843)$ & 0.204 \\
RP + PLND-laparascopic & 0.002 \\
RP + PLND-robotic & & \\
\hline
\end{tabular}

ADT indicates androgen deprivation therapy; $\mathrm{BT}$, brachytherapy; $\mathrm{Cl}$, confidence interval controlled for program, facility, geography, income, age comorbidity score, clinical T stage, clinical Gleason score, PSA, and treatment delay; EBRT, external beam radiotherapy; RP, radical prostatectomy; RT, radiotherapy.

and race on survival, and stratified analysis demonstrated black race was a more negative prognosticator in age younger than 60 compared with age older than 60 (Table A8, Supplemental Digital Content 8, http://links.lww.com/IJSO/A12).

\section{Discussion}

Despite the abundance of previous evidence suggesting black men have a more aggressive prostate cancer compared with white men, our data introduces significant areas of research still to be explored.

In our cohort, race was a significant variable among LR patients, with superior survival observed in white men. Notably, this cohort did not include patients that remained on AS for the entire duration of the study as all patients in this study received definitive therapy, and our PS-MVA controlled for whether patients received treatment within 90 days of diagnosis, so delay of treatment is unlikely to explain this difference in survival. In addition, our PS-MVA controlled for socioeconomic factors including income, geography, and treatment facility, thus we

\section{Table 4}

Sensitivity analysis stratifying unfavorable risk cohort receiving surgery by adjuvant treatment.

\begin{tabular}{lcr}
\hline Variables & HR $\mathbf{( 9 5 \%} \mathbf{~ C l})$ & $\boldsymbol{P}$ \\
\hline Race & Reference & \\
$\quad$ Black & $0.949(0.856-1.053)$ & 0.324 \\
White & & \\
Treatment & Reference & \\
EBRT + ADT & $0.756(0.668-0.856)$ & $<0.001$ \\
EBRT + BT + ADT & $0.642(0.520-0.792)$ & $<0.001$ \\
RP + ADT & $0.469(0.421-0.522)$ & $<0.001$ \\
RP & $0.899(0.731-1.107)$ & 0.316 \\
RP + RT + ADT & $0.738(0.599-0.909)$ & 0.004 \\
RP + RT & & \\
\hline
\end{tabular}

ADT indicates androgen deprivation therapy; $\mathrm{BT}$, brachytherapy; $\mathrm{Cl}$, confidence interval controlled for program, facility, geography, income, age comorbidity score, clinical T stage, clinical Gleason score, PSA, and treatment delay; EBRT, external beam radiotherapy; RP, radical prostatectomy; RT, radiotherapy. 
Table 5

Sensitivity analysis stratifying unfavorable risk cohort by surgical approach.

\begin{tabular}{|c|c|c|}
\hline Variables & HR (95\% Cl) & $P$ \\
\hline \multicolumn{3}{|l|}{$\overline{\text { Race }}$} \\
\hline Black & Reference & \\
\hline White & $0.942(0.849-1.040)$ & 0.256 \\
\hline \multicolumn{3}{|l|}{ Treatment } \\
\hline EBRT + ADT & Reference & \\
\hline RP-laparascopic & $0.618(0.292-1.31)$ & 0.207 \\
\hline RP-robotic & $0.616(0.487-0.777)$ & $<0.001$ \\
\hline$E B R T+B T+A D T$ & $0.757(0.669-0.857)$ & $<0.001$ \\
\hline
\end{tabular}

ADT indicates androgen deprivation therapy; $\mathrm{BT}$, brachytherapy; $\mathrm{Cl}$, confidence interval controlled for program, facility, geography, income, age comorbidity score, clinical T stage, clinical Gleason score, PSA, and treatment delay; EBRT, external beam radiotherapy; RP, radical prostatectomy; RT, radiotherapy.

posit that the observed survival difference may be attributed to differences in disease biology between white and black men. Our current risk stratification may be failing to appropriately stage early prostate cancer in black men who may not actually have favorable risk disease.

Such a difference in biology is consistent with several retrospective analyses, demonstrating that black men are at increased risk of upstaging which reflects a more aggressive disease process. In the Johns Hopkins active surveillance registry, black men clinically categorized as very LR had an increased risk of upstaging after RP compared with other races ${ }^{[1]}$. The group also found black men were more likely to be upstaged in serial biopsy based on grade rather than volume of disease, suggesting the need for a different criteria for selecting appropriate black men for active surveillance ${ }^{[12]}$. Another series of 272 patients on active surveillance found black race to be a significant predictor for upstaging on active surveillance after a median follow-up time of 2.9 years with a mean of 2.3 surveillance biopsies ${ }^{[13]}$. Similarly, a series from the University of Miami found black race to be a significant predictor for upstaging risk on $\mathrm{AS}^{[13]}$, while a series from Duke showed that black race significantly predicted for discontinuation of AS, reflecting disease progression ${ }^{[14]}$.

Current guidelines recommend that very low risk, LR, and favorable intermediate risk prostate cancer patients be considered for AS consisting of PSA testing every 6 months, digital rectal exam and biopsy annually ${ }^{[15]}$. Although AS prevents overtreatment of patients at LR of disease progression, many of the studies establishing the basis of this treatment paradigm were based on prior studies that underrepresented minorities ${ }^{[16]}$, particularly black men who are known to have significantly lower prostate cancer-specific survival rates compared with all other races.

It is important to note however that our study found that income bracket was the only socioeconomic variable to significantly interact with race on survival in the favorable cohort, which was further confirmed in the subgroup analysis. The significant interaction suggests there may be underlying socioeconomic variables not adequately captured in the NCDB which may contribute to the survival difference noted, and an exclusively biological explanation for the noted differences may not be completely accurate either.

Given that we found significant interaction between age and race on survival in the unfavorable risk cohort, we conducted a subgroup analysis using the age cut off of 60 as used in prior studies ${ }^{[17,18]}$ to distinguish between "young" and "old" patients, in order to investigate weather race influenced outcomes differently in younger versus older cohorts receiving risk-appropriate definitive surgical resection or radiation therapy. Our analysis showed that race had no significant prognostic role in unfavorable risk patients, regardless of age.

A recent pooled retrospective study compared prostate cancerspecific mortality (PCSM) in patients with nonmetastatic prostate adenocarcinoma (cT1-4N0-1M0) using cases from SEER, the Veterans Affairs health system and four randomized clinical trial cohorts. Similar to our findings, the study demonstrated a stepwise reduction in a survival difference between black and white men after controlling for age, stage and socioeconomic variables, and demonstrated a lack of survival difference between the 2 races among the high risk subgroup. Interestingly however, black men had improved PCSM compared with white men in the cohort originating from the 4 randomized trials among all patients and in the high risk subgroup ${ }^{[19]}$. While our study did not demonstrate a survival advantage in black men, a significant interaction was found with age and race in the unfavorable risk cohort, with an increase in the HR for white race (reference black race) from age younger than $60(\mathrm{HR}, 0.862)$ to age older than $60(\mathrm{HR}$, $1.070)$, which suggests that young black men with prostate cancer may have a more aggressive prostate cancer that is biologically distinct entity from that of young white men.

Notably, our study only included patients who received NCCN recommended standard of care treatments and could be adequately risk stratified. In particular, patients receiving radiation must have received adequate radiation doses (at least $7560 \mathrm{cGy}$ ), and unfavorable risk patients receiving EBRT must have received ADT as well. In addition, unfavorable risk patients receiving RP must have received a PLND. Our models also controlled for the interval between diagnosis to treatment initiation, and propensity matching ensured well-balanced cohorts to eliminate any treatment-related or socioeconomic imbalances between black and white men. Finally, our study is the first to observe the role of race on survival in different risk cohorts using the NCDB.

Unfortunately, the NCDB only records OS and not PCSM or biochemical recurrence-free survival, which are more meaningful endpoints in prostate cancer research. In addition, because of the nature of retrospective studies, our analysis is limited by a lack of randomization which could account for both known and unknown confounders. By carrying out a propensity-matched analysis, we were able to mimic the randomization process as closely as possible, but our model remains limited to the known variables available in the database. Although we created homogenized cohorts for comparisons in all our models, we inevitably lost observations by doing so, which may inadvertently introduce a selection bias. Therefore, our conclusions are only meant to be hypothesis generating.

\section{Conclusions}

Black men, while shown to be a negative prognosticator in patients with favorable risk prostate cancer, did not predict for worse OS in unfavorable risk disease, an observation made independent of socioeconomic and demographic factors. This suggests that the biology of disease plays a significant role in favorable risk cohorts, but in those with more advanced higher 
risk disease, these race-related biological differences carry no significant influence.

\section{Ethical approval}

NewYork-Presbyterian Brooklyn Methodist Hospital IRB.

\section{Sources of funding}

The authors received no specific funding for this work.

\section{Author contribution}

All authors listed have contributed sufficiently to the project to be included as authors, and all those who are qualified to be authors are listed in the author byline.

\section{Conflicts of interest disclosure}

The authors declare that they have no financial conflict of interest with regard to the content of this report.

\section{Research registration unique identifying number (UIN)}

IRB approval ID\#1241936.

\section{Guarantor}

NewYork-Presbyterian Brooklyn Methodist Hospital.

\section{Acknowledgments}

The National Cancer Data Base (NCDB) is a joint project of the Commission on Cancer ( $\mathrm{CoC}$ ) of the American College of Surgeons and the American Cancer Society. The CoC's NCDB and the hospitals participating in the CoC NCDB are the source of the deidentified data used herein; they have not verified and are not responsible for the statistical validity of the data analysis or the conclusions derived by the authors.

\section{References}

[1] Sundi D, Ross AE, Humphreys EB, et al. African American men with very low-risk prostate cancer exhibit adverse oncologic outcomes after radical prostatectomy: should active surveillance still be an option for them? J Clin Oncol 2013;31:2991-7.
[2] Tsivian M, Bañez LL, Keto CJ, et al. African-American men with lowgrade prostate cancer have higher tumor burdens: results from the Duke Prostate Center. Prostate Cancer Prostatic Dis 2013;16:91-4.

[3] Yamoah K, Johnson MH, Choeurng V, et al. Novel biomarker signature that may predict aggressive disease in African American men with prostate cancer. J Clin Oncol 2015;33:2789-96.

[4] Powell IJ, Bock CH, Ruterbusch JJ, et al. Evidence supports a faster growth rate and/or earlier transformation to clinically significant prostate cancer in black than in white American men, and influences racial progression and mortality disparity. J Urol 2010;183:1792-6.

[5] Evans S, Metcalfe C, Patel B, et al. Clinical presentation and initial management of black men and white men with prostate cancer in the United Kingdom: the PROCESS cohort study. Br J Cancer 2010;102: 249-54.

[6] Faisal FA, Sundi D, Cooper JL, et al. Racial disparities in oncologic outcomes after radical prostatectomy: long-term follow-up. Urology 2014;84:1434-41.

[7] Moul JW, Sesterhenn IA, Connelly RR, et al. Prostate-specific antigen values at the time of prostate cancer diagnosis in African-American men. JAMA 1995;274:1277-81.

[8] Sanchez-Ortiz RF, Troncoso P, Babaian RJ, et al. African-American men with nonpalpable prostate cancer exhibit greater tumor volume than matched white men. Cancer 2006;107:75-82.

[9] Friedlander DF, Trinh Q-D, Krasnova A, et al. Racial disparity in delivering definitive therapy for intermediate/high-risk localized prostate cancer: the impact of facility features and socioeconomic characteristics. Eur Urol 2017;73:445-51.

[10] Austin PC. Balance diagnostics for comparing the distribution of baseline covariates between treatment groups in propensity-score matched samples. Stat Med 2009;28:3083-107.

[11] Coughlin GD, Yaxley JW, Chambers SK, et al. Robot-assisted laparoscopic prostatectomy versus open radical retropubic prostatectomy: 24month outcomes from a randomised controlled study. Lancet Oncol 2018;19:1051-60.

[12] Sundi D, Faisal FA, Trock BJ, et al. Reclassification rates are higher among African American men than Caucasians on active surveillance. Urology 2015;85:155-60.

[13] Iremashvili V, Soloway MS, Rosenberg DL, et al. Clinical and demographic characteristics associated with prostate cancer progression in patients on active surveillance. J Urol 2012;187:1594-9.

[14] Abern MR, Bassett MR, Tsivian M, et al. Race is associated with discontinuation of active surveillance of low-risk prostate cancer: results from the Duke Prostate Center. Prostate Cancer Prostatic Dis 2013;16: 85-90.

[15] Carroll PH, Mohler JL. NCCN guidelines updates: prostate cancer and prostate cancer early detection. J Natl Compr Canc Netw 2018; 16:620-3.

[16] Tosoian JJ, Trock BJ, Landis P, et al. Active Surveillance Program for Prostate Cancer: an Update of the Johns Hopkins Experience. J Clin Oncol 2011;29:2185-90.

[17] Klayton TL, Ruth K, Horwitz EM, et al. Young age under 60 years is not a contraindication to treatment with definitive dose escalated radiotherapy for prostate cancer. Radiother Oncol 2011;101:508-12.

[18] Ashamalla H, Guirguis A, McCool K, et al. Brachytherapy improves outcomes in young men ( $\leq 60$ years) with prostate cancer: a SEER analysis. Brachytherapy 2017;16:323-9.

[19] Dess RT, Hartman HE, Mahal BA, et al. Association of black race with prostate cancer-specific and other-cause mortality. JAMA Oncology 2019;5:975-83. 